\title{
Weakly nonlinear ac response: Theory and application
}

\author{
Zhong-shui Ma \\ Department of Physics, The University of Hong Kong, Pokfulam Road, Hong Kong, China \\ and Advanced Research Center, Zhongshan University, Guangzhou, China \\ Jian Wang \\ Department of Physics, The University of Hong Kong, Pokfulam Road, Hong Kong, China \\ Hong Guo \\ Department of Physics, The University of Hong Kong, Pokfulam Road, Hong Kong, China \\ and Center for the Physics of Materials and Department of Physics, McGill University, Montreal, PQ, Canada H3A 2T8
}

(Received 17 September 1998)

\begin{abstract}
We report a microscopic and general theoretical formalism for electrical response, which is appropriate for both dc and ac weakly nonlinear quantum transport. The formalism emphasizes the fundamental requirements of current conservation and gauge invariance. It makes a formal connection between linear response and scattering matrix theory at the weakly nonlinear level. We present three examples of application of this formalism to analyze the dynamic conductance and the nonlinear-nonequilibrium charge distribution for multiprobe coherent quantum conductors. [S0163-1829(99)06411-5]
\end{abstract}

Over the past decade tremendous efforts have been devoted in developing quantum transport theories that are appropriate for quantum coherent conductors. ${ }^{1}$ While substantial progress has been achieved, so far there is still a lack of a general formalism that works not only in the linear dc regime but also in both $\mathrm{dc}$ and ac nonlinear regimes. ac transport involves time-dependent fields, thus, induction is important as characterized by, e.g., the existence of displacement current. Many transport theories do not consider this ingredient, resulting in a violation of current conservation as pointed out by Büttiker and Christen. ${ }^{2}$ The nonlinear $\mathrm{dc}$ transport coefficients appear in front of powers of external bias voltage in the expression of electric current: $I$ $=I\left(\left\{V_{\alpha}\right\}\right)$, where $V_{\alpha}$ is the potential at a probe labeled $\alpha$. A correct theory must maintain gauge invariance in addition to the current conservation: the physics should not change when potential at the probes are all shifted by the same constant amount. ${ }^{2}$

So far, current conserving and gauge invariant quantum transport has been analyzed using the scattering matrix theory (SMT) ${ }^{2-4}$ Electron-electron (e-e) interaction was found to play the essential role in obtaining these fundamental requirements. In $\mathrm{SMT}^{2}$ various ac frequency $\omega$-independent partial density of states (PDOS) appear naturally, which characterizes the scattering. The SMT is quite intuitive and can be implemented numerically for practical calculations. ${ }^{5}$ However, so far no theoretical formalism exists that can systematically go beyond the linear- $\omega$ ac and second-order dc transport coefficients, and that satisfies current conservation and gauge invariance. There are also no approaches available to analyze the weakly nonlinear ac transport. Hence, up to now, these important problems have not been investigated systematically. It is well known that the linear transport can be discussed using either SMT or using linear response. ${ }^{6,7}$ In the linear dc regime, connection of these two approaches has been formalized ${ }^{7}$ and attempt has been made to generalize it to nonlinear dc situation. ${ }^{8}$ It is the purpose of this paper to report a general theoretical formalism derivable from the response theory which is appropriate for both dc and ac weakly nonlinear regimes in addition to linear situations.

Before going into the details, we summarize the main results: (1) A general formalism for deriving ac and dc nonlinear transport coefficients has been found, which is current conserving and gauge invariant. (2) The formalism is put into a form that is numerically calculable for mesoscopic or even microscopic conductors order by order in weak bias and in $\omega .{ }^{9}$ (3) The results obtained from SMT are reproduced in this formalism, thus we make the formal connection between SMT and the response theory at the weakly nonlinear dc and ac level. (4) Generalized notions of the characteristic potential, the nonequilibrium charge, and the Lindhard functions are derived naturally from the time-dependent internal response. (5) We give three examples for applications of this formalism.

A response theory considers a time-dependent perturbation $^{7} H^{\prime} \equiv V(\mathbf{r}, t)=V(\mathbf{r}) e^{-\delta|t|} \cos (\Omega t)$ to the Hamiltonian $H_{o}$ of a conductor. Here, $H_{o}$ contains the kinetic energy, the influence of an external static magnetic field, the confining potential of the conductor, or any other static potential. We assume that the problem of $H_{o}$ has been solved, $H_{o} \psi_{n}=\epsilon_{n} \psi_{n}$, and we are interested in the effect of $H^{\prime}$. It is crucial to realize that just solving the problem of $H_{o}+H^{\prime}$ will not generate a current conserving and gauge invariant transport theory, since the time-varying field induces an internal potential $^{2} U(t)$ and a vector potential $\mathbf{A}(t)$, which must be included into the analysis. The reason one has to consider $\mathbf{A}(t)$ is due to the Ampere-Maxwell's law and Faraday's law. It is these internal potentials that generate such effect as a displacement current. Mathematically, one needs to solve a quantum-mechanical problem in conjunction with the Maxwell equations. We thus start from a self-consistent 
(up to the Hartree level) Hamiltonian $H$ coupled with the Helmholtz equation in the Lorentz gauge

$$
\begin{gathered}
H=H_{o}+H^{\prime}+e U-\frac{e \mathbf{A}}{2 m c} \cdot\left(\mathbf{p}-\frac{e \mathbf{A}_{0}}{c}\right) \\
-\left(\mathbf{p}-\frac{e \mathbf{A}_{0}}{c}\right) \cdot \frac{e \mathbf{A}}{2 m c}+\frac{e^{2} A^{2}}{2 m c^{2}} \\
\nabla^{2} U(\mathbf{r}, t)-\partial_{t}^{2} U(\mathbf{r}, t)=-4 \pi \delta \rho(\mathbf{r}, t), \\
\nabla^{2} \mathbf{A}(\mathbf{r}, t)-\partial_{t}^{2} \mathbf{A}(\mathbf{r}, t)=-4 \pi \delta \mathbf{J}(\mathbf{r}, t),
\end{gathered}
$$

where $\delta \rho$ is the total charge involved in the response including those of the induced and $\delta \mathbf{J}$ is the total current density, $\mathbf{A}_{0}$ is the vector potential for the static external magnetic field. Atomic units will be used in the following by setting $\hbar=c=e=2 m=1$.

We shall be interested in a multiprobe conductor small enough such that quantum coherence is maintained. Inside a probe $\alpha$ far away from the scattering region, the amplitude of the external disturbance is written as $V_{\alpha}$. Since there is no hope of solving the above equations exactly, we shall calculate $U(\mathbf{r}, t)$ and $\mathbf{A}(\mathbf{r}, t)$ in a series form, expanded in powers of $V_{\alpha}$ using the characteristic potentials ${ }^{2}$ (CP) $u_{\alpha}(\mathbf{r}, t), u_{\alpha \beta}(\mathbf{r}, t) \ldots$ and the characteristic vector potential $(\mathrm{CVP}) \mathbf{a}_{\alpha}(\mathbf{r}, t)$ and $\mathbf{a}_{\alpha \beta}(\mathbf{r}, t) \ldots$,

$$
\begin{aligned}
& U=\sum_{\alpha} u_{\alpha} V_{\alpha}+\frac{1}{2} \sum_{\alpha \beta} u_{\alpha \beta} V_{\alpha} V_{\beta}+\cdots \\
& \mathbf{A}=\sum_{\alpha} \mathbf{a}_{\alpha} V_{\alpha}+\frac{1}{2} \sum_{\alpha \beta} \mathbf{a}_{\alpha \beta} V_{\alpha} V_{\beta}+\cdots .
\end{aligned}
$$

These expansions make sense at weakly nonlinear regime. The CP and CVP, respectively, characterize the system response to an external time-dependent perturbation. The physics associated with weakly nonlinear quantum transport is related to the CP's and CVP's of the appropriate order (see below). Gauge invariance puts constraints on the $\mathrm{CP}$ and CVP, for instance $\quad \Sigma_{\alpha} u_{\alpha}(\mathbf{r}, t)=1, \quad \Sigma_{\alpha} u_{\alpha \beta}(\mathbf{r}, t)$ $=\Sigma_{\beta} u_{\alpha \beta}(\mathbf{r}, t)=0$, and in general $\Sigma_{\gamma \in \beta} u_{\alpha\{\beta\}_{l}}=0$. For CVP, we have $\Sigma_{\gamma \in \alpha} \mathbf{a}_{\{\alpha\}_{l}}=0$. Here, the subscript $\{\beta\}_{l}$ is a short notation of $l$ indices $\gamma, \delta, \eta, \cdots$.

The quantum-mechanics problem of Eq. (1) is solved in the standard series fashion by iterating the Liouville-von Neumann equation for the density operator: ${ }^{10} d \hat{\rho} / d t$ $=-i[H, \hat{\rho}]$. In this procedure we expand both $H$ and $\hat{\rho}$ into series form order by order in the external perturbation strength: $\hat{\rho}=\hat{\rho}_{0}+\Sigma_{l=1} \hat{\rho}_{l} ; \quad H=H_{o}+\Sigma_{l=1} H_{l}$. Using Eqs. (1), (4), and (5), $H_{l}$ is easily derived in terms of CP's and CVP's. From the Liouville-von Neumann equation, order by order we thus derive equations for the terms $\hat{\rho}_{l}$. These equations can be formally solved in terms of the CP's and CVP's at the appropriate order: $\hat{\rho}_{1}$ is related to $u_{\alpha}$ and $\mathbf{a}_{\alpha} ; \hat{\rho}_{2}$ is related to $u_{\alpha \beta}, u_{\alpha}, \mathbf{a}_{\alpha}$, and $\mathbf{a}_{\alpha \beta}$; etc. For example, the lowest order term is $\hat{\rho}_{1}=-i U_{o} \int_{-\infty}^{t} d t_{1}\left[H_{1}^{\prime}\left(t_{1}\right), \hat{\rho}_{o}\right] U_{o}^{\dagger}$, where $\quad H_{1}^{\prime} \equiv U_{o}^{\dagger} H_{1} U_{o}, U_{o} \equiv \exp \left(-i H_{o} t\right), \quad$ and $\hat{\rho}_{o}$ $=\Sigma_{n} f\left(\epsilon_{n}\right)|n\rangle\langle n|$ is the equilibrium density matrix. These formal expressions of $\hat{\rho}_{l}$ can be written in the frequency space to explicitly see the $\omega$ dependence. We then formally evaluate the matrix elements $\left(\hat{\rho}_{l}\right)_{m n}=\left\langle m\left|\hat{\rho}_{l}\right| n\right\rangle$ using the basis set $\psi_{n}$. This gives, for example, $\left(\hat{\rho}_{1}\right)_{m n}=f_{m n}$ $\left[\left(V-U_{1}\right)_{m n}+i \mathbf{A}_{1} \cdot \mathbf{W}_{m n}\right] /\left(\boldsymbol{\epsilon}_{n m}+\omega+i \eta\right)$. Here, $U_{1}$ and $\mathbf{A}_{1}$ is the first term on the right-hand side of Eqs. (4) and (5), respectively; and $\mathbf{W}_{n m}(\mathbf{r}) \equiv \psi_{n}^{*}(\mathbf{r}) \overleftrightarrow{\mathcal{D}} \psi_{m}(\mathbf{r})$ with $\overleftrightarrow{\mathcal{D}}$ the standard double-sided derivative operator; ${ }^{7} \epsilon_{n m} \equiv \epsilon_{n}-\epsilon_{m}, f_{n m}$ $\equiv f\left(\epsilon_{n}\right)-f\left(\epsilon_{m}\right)$, and $\eta$ is infinitesimal. Higher order terms can be derived in similar fashion but to save room we leave them out of this paper. Using the matrix elements we can, still formally, derive the expression for the charge and current density distribution, ${ }^{10}$ which enter the right-hand side of the Helmholtz Eqs. (2) and (3): $\quad \delta \rho(\mathbf{r}, t)=\Sigma_{l} \Sigma_{m n}\left(\hat{\rho}_{l}\right)_{m n} \psi_{n}^{*} \psi_{m} \quad$ and $\quad \delta \mathbf{J}(\mathbf{r}, t)=$ $-\Sigma_{l} \Sigma_{m n}\left(\hat{\rho}_{l}\right)_{m n}\left(i \mathbf{W}_{n m}-\mathbf{A} \psi_{m}^{*} \psi_{n}\right)$. This makes Eqs. (2) and (3) into a solvable form.

With the charge and current density distributions, from Eqs. (2) and (3), we can derive equations for the CP's and CVP's. This is one of the central results of this work

$$
\begin{aligned}
& -\nabla^{2} u_{\{\alpha\}_{l}}(\mathbf{r}, \omega)-\omega^{2} u_{\{\alpha\}_{l}}(\mathbf{r}, \omega)=4 \pi \delta \rho_{\{\alpha\}_{l}}(\mathbf{r}, \omega) \\
& -\nabla^{2} \mathbf{a}_{\{\alpha\}_{l}}(\mathbf{r}, \omega)-\omega^{2} \mathbf{a}_{\{\alpha\}_{l}}(\mathbf{r}, \omega)=4 \pi \delta \mathbf{J}_{\{\alpha\}_{l}}(\mathbf{r}, \omega),
\end{aligned}
$$

where the nonlinear charge density $\delta \rho$ is given by

$$
\begin{aligned}
\delta \rho_{\{\alpha\}_{l}}(\mathbf{r}, \omega)= & \frac{d n_{\{\alpha\}_{l}}(\mathbf{r}, \omega)}{d \epsilon}-\int d \mathbf{r}_{1} \Pi\left(\mathbf{r}, \mathbf{r}_{1}, \omega\right) u_{\{\alpha\}_{l}}\left(\mathbf{r}_{1}, \omega\right) \\
& -\int d \mathbf{r}_{1} \mathbf{F}\left(\mathbf{r}, \mathbf{r}_{1}, \omega\right) \cdot \mathbf{a}_{\{\alpha\}_{l}}\left(\mathbf{r}_{1}, \omega\right)
\end{aligned}
$$

The current density is given by $\delta \mathbf{J}_{\{\alpha\}_{l}}(\mathbf{r}, \omega)$ $=-i \Sigma_{m n}\left(\delta \rho_{\{\alpha\}_{l}}\right)_{n m} \mathbf{W}_{m n}+\Sigma_{m n} \Sigma_{k}\left(\delta \rho_{\{\alpha\}_{l-k}}\right)_{m n} \mathbf{a}_{\{\alpha\}_{k}} \psi_{m}^{*} \psi_{n} / k !$ with $l>k$. And $\left(\delta \rho_{\{\alpha\}_{l}}\right)_{m n}$ is obtained from the densitymatrix element: $\left(\hat{\rho}_{l}\right)_{m n} \equiv \Sigma_{\gamma \in \alpha}\left(\delta \rho_{\{\alpha\}_{l}}\right)_{m n} V_{\{\alpha\}_{l}}$ with $V_{\{\alpha\}_{l}}$ $=V_{\alpha} V_{\beta} \ldots$.

The first term of the right-hand side of Eq. (8) is given by the frequency-dependent local density of states (LDOS), which can be derived using the Green's functions. For example the lowest order (one index) LDOS is given by

$$
\frac{d n_{\alpha}(\mathbf{r}, \omega)}{d \boldsymbol{\epsilon}}=-\int d y_{1 \alpha} \sum_{m n} \frac{f_{n m}}{\epsilon_{n m}} \frac{\psi_{n}^{*}(\mathbf{r}) \psi_{m}(\mathbf{r}) \mathbf{W}_{m n}\left(\mathbf{r}_{1}\right) \cdot \hat{\mathbf{x}}_{1 \alpha}}{\epsilon_{n m}+\omega+i \eta} .
$$

The integration in Eq. (9) is along the boundary of probe $\alpha$, and unit vector $\hat{\mathbf{x}}_{1 \alpha}$ is the direction along this probe. This expression can be further written in terms of the Green's function. The second term on the right of Eq. (8) is the induced charge written in terms of a frequency-dependent Lindhard function, ${ }^{11}$ which is given by

$$
\Pi\left(\mathbf{r}, \mathbf{r}_{1}, \omega\right)=\sum_{m n} \frac{f_{n m} \psi_{n}^{*}(\mathbf{r}) \psi_{m}(\mathbf{r}) \psi_{m}^{*}\left(\mathbf{r}_{1}\right) \psi_{n}\left(\mathbf{r}_{1}\right)}{\epsilon_{n m}+\omega+i \eta} .
$$

The third term on the right of Eq. (8) is the frequencydependent response function for the induced vector potential 


$$
\mathbf{F}\left(\mathbf{r}, \mathbf{r}_{1}, \omega\right)=i \sum_{m n} \frac{f_{n m} \psi_{n}^{*}(\mathbf{r}) \psi_{m}(\mathbf{r}) \mathbf{W}_{m n}\left(\mathbf{r}_{1}\right)}{\boldsymbol{\epsilon}_{n m}+\omega+i \eta}
$$

Some discussions of Eqs. (6) and (7) are needed. (1) The characteristic potentials $\mathrm{CP}$ and CVP have multiple indices that are necessary in order to study nonlinear (in bias) transport coefficients. (2) The concept of LDOS is generalized to higher order: at linear order (one index) it is the frequencydependent injectivity, ${ }^{3}$ while at higher order (more than one indices) it also contains some internal response of the lower order. While their expressions [not shown except in Eq. (9)] are complicated, they are all expressed in terms of the Green's functions. (3) It can be shown that the Lindhard function is related to the first-order (one index) total LDOS, $\int d \mathbf{r}_{1} \Pi\left(\mathbf{r}, \mathbf{r}_{1}, \omega\right)=d n(\mathbf{r}, \omega) / d \epsilon, \quad$ where $\quad d n(\mathbf{r}, \omega) / d \epsilon$ $=\Sigma_{\alpha} d n_{\alpha}(\mathbf{r}, \omega) / d \epsilon$. (4) After solving the CP and CVP from Eqs. (6) and (7), we obtain the density-matrix elements $\left(\hat{\rho}_{l}\right)_{m n}$ at the appropriate order. This allows the explicit calculations of the charge and current distribution $\delta \rho(\mathbf{r}, \omega)$ and $\delta \mathbf{J}(\mathbf{r}, \omega)$ using the expressions given above. Various transport properties can be obtained immediately.

Nonlinear "Capacitance." From the total charge distribution, we can write

$$
Q_{\alpha}=\sum_{\beta} C_{\alpha \beta} V_{\beta}+1 / 2 \sum_{\beta \gamma} C_{\alpha \beta \gamma} V_{\beta} V_{\gamma}+\cdots,
$$

where $Q_{\alpha}$ is just the appropriate spatial integration of the charge density. Hence, the nonlinear theory naturally allows the definition of nonlinear capacitance coefficients,

$$
C_{\{\alpha\}_{l}}(\omega)=\int_{\Gamma} d \mathbf{r} \delta \rho_{\{\alpha\}_{l-1}}(\mathbf{r}, \omega),
$$

where index $l>1$. The spatial integral over $\Gamma$ means integrating over the region, where the charge $Q$ is positive (or negative). At linear order in both $\omega$ and voltage, i.e., when $l=2$ and letting $\omega=0$, this gives ${ }^{12}$ the electrochemical capacitance, ${ }^{3}$ which is of great experimental interest. ${ }^{13}$ Note that due to the finite screening length resulting from small DOS for mesoscopic conductors, a nonequilibrium charge distribution can be established even when there is dc coupling between the two capacitor "plates" (the $+Q$ and $-Q$ regions). ${ }^{14}$ If we keep the general $\omega$ dependence but still work on the linear bias order $(l=2)$, Eq. (13) gives the general linear dynamic response of the charge. A further very interesting result of Eq. (13) is the nonlinear "capacitance" for $l>2$ and setting $\omega=0$. Such a quantity does not seem to have a geometrical counterpart, and it measures the degree of the nonequilibrium charge pile-up at the nonlinear order.

In particular, let's calculate $C_{\alpha \beta \gamma}$ for a parallel plate capacitor, where each plate has an area $A$ and is infinitely thin, and they are located in space on the $y-z$ plane at positions $x=0$ and $x=a$. Using Eq. (6) to solve the characteristic potentials at different regions from $x=-\infty$ to $x=+\infty$, matching the solutions at $x=0, a$, we obtain $u_{1}$ and $u_{11}$. Within the Thomas-Fermi approximation of the Lindhard function, from Eq. (13) we obtain

$$
2 \frac{C_{111}}{C_{11}^{3}}=\frac{d}{d \epsilon}\left[\left(\frac{d N_{2}}{d \epsilon}\right)^{-2}-\left(\frac{d N_{1}}{d \epsilon}\right)^{-2}\right],
$$

where $d N_{i} / d \epsilon$ is the total DOS on plate $i$, and $C_{11}$ $=\left[4 \pi a / A+\sum_{i=1}^{2} 1 /\left(d N_{i} / d \epsilon\right)\right]^{-1}$ is the usual electrochemical capacitance. ${ }^{3}$ This scaling relation gave us an extra handle on the microscopics of a conductor: by measuring capacitances and forming the scaling combination, all that is left is the energy derivatives of DOS. We have checked that for another system, being two large metallic rods with their ends at a distance $a$ apart, exactly the same scaling of $C_{111} / C_{11}^{3}$ is obtained, the only difference being that the factor 2 on the left side changes to 6. Equation (14) suggests an interesting experiment by using two mesoscopic conductors that couple capacitively, and one can study the DOS of the tiny conductors by measuring the ac response as a function of the amplitude of the ac bias.

Linear Dynamic Conductance. As a second example we derive the dynamic conductance $G_{\alpha \beta}(\omega) . G_{\alpha \beta}$ is defined by the electric current flowing through the probe $\alpha$ : $\mathbf{I}_{\alpha}^{(1)}$ $=\Sigma_{\beta} G_{\alpha \beta}(\omega) V_{\beta} . \quad \mathbf{I}_{\alpha}^{(1)}$ is obtained by a spatial integration of the current density across the transverse direction of the probe, where the expression for current density has been given above. We obtain,

$$
\begin{aligned}
G_{\alpha \beta}(\omega)= & G_{\alpha \beta}(0)-i \omega\left\{\int d \epsilon\left(-\partial_{\epsilon} f\right) \frac{d N_{\alpha \beta}(\omega)}{d \epsilon}\right. \\
& -\int d \mathbf{r}\left[\frac{d \bar{n}_{\alpha}(\mathbf{r}, \omega)}{d \epsilon} u_{\beta}(\mathbf{r}, \omega)\right. \\
& \left.\left.+\frac{d \overline{\mathbf{M}}_{\alpha}(\mathbf{r}, \omega)}{d \epsilon} \cdot \mathbf{a}_{\beta}(\mathbf{r}, \omega)\right]\right\}
\end{aligned}
$$

where

$$
\frac{d \mathbf{M}_{\alpha}(\mathbf{r}, \omega)}{d \epsilon}=-\int d y_{1 \alpha} \sum_{m n} \frac{f_{n m} \mathbf{W}_{n m}\left(\mathbf{r}_{1}\right) \cdot \hat{\mathbf{x}}_{1 \alpha} \mathbf{W}_{m n}}{\boldsymbol{\epsilon}_{n m}+\omega+i \eta} .
$$

Here, $G_{\alpha \beta}(0)$ is the familiar linear dc conductance that can be calculated using the transmission coefficient. ${ }^{7}$ $d \bar{n}_{\alpha}(\mathbf{r}, \omega) / d \epsilon$ is another LDOS dual of $d n_{\alpha}(\mathbf{r}, \omega) / d \epsilon$ which is obtained by substitution $\omega \rightarrow-\omega$ and $\eta \rightarrow-\eta$ in Eq. (9). $d \overline{\mathbf{M}}_{\alpha}(\mathbf{r}, \omega) / d \epsilon$ is dual of $d \mathbf{M}_{\alpha}(\mathbf{r}, \omega) / d \epsilon$. Keeping the firstorder term in $\omega$ and neglecting the contribution from the CVP, this result reduces exactly to the emittance obtained by SMT. ${ }^{2,3}$ The $\omega$-dependent parts are given as a sum of two contributions. The first is due to the external perturbation at a reservoir, and it is determined by the $\omega$-dependent total PDOS $d N_{\alpha \beta}(\omega) / d \epsilon$. The second is due to induction and is determined by the internal response. It is not difficult to verify $\Sigma_{\alpha} G_{\alpha \beta}(\omega)=0$.

Weakly Nonlinear Dynamic Conductance. Our theory can be used to analyze weakly nonlinear ac transport to higher order in bias, and we have derived the second-order expression defined by the second-order electric current $\mathbf{I}_{\alpha}^{(2)}$ 
$=\sum_{\beta \gamma} G_{\alpha \beta \gamma}(\omega) V_{\beta} V_{\gamma} . \quad \mathbf{I}_{\alpha}^{(2)}$ is calculated in similar fashion as the $\mathbf{I}_{\alpha}^{(1)}$ of the last example using the expression given above. The final result is

$$
\begin{aligned}
G_{\alpha \beta \gamma}(\omega)= & G_{\alpha \beta \gamma}(0)-\frac{i}{2} \omega\left\{\int d \epsilon\left(-\partial_{\epsilon} f\right) \frac{d N_{\alpha \beta \gamma}(\omega)}{d \epsilon}\right. \\
& -\int d \mathbf{r}\left(\frac{d \bar{n}_{\alpha}(\mathbf{r}, \omega)}{d \epsilon} u_{\beta \gamma}(\mathbf{r}, \omega)\right. \\
& \left.\left.+\frac{d \overline{\mathbf{M}}_{\alpha}(\mathbf{r}, \omega)}{d \epsilon} \cdot \mathbf{a}_{\beta \gamma}(\mathbf{r}, \omega)\right)\right\}
\end{aligned}
$$

In this result, $G_{\alpha \beta \gamma}(0)$ is the second-order weakly nonlinear dc conductance, which was studied using SMT. ${ }^{2}$ The current conservation and gauge invariance can be explicitly confirmed: $\Sigma_{\gamma} G_{\alpha \beta \gamma}(\omega)=0 ; \quad \Sigma_{\alpha} G_{\alpha \beta \gamma}(\omega)=\Sigma_{\beta} G_{\alpha \beta \gamma}(\omega)=0$.

Keeping terms linear in $\omega$, from Eq. (17) we derive the dynamic conductance to the order $\omega V^{2}$. For the example of double-barrier resonant tunneling device, near a resonance energy $E \sim E_{r}$, the scattering matrix takes the Breit-Wigner form. This allows simple expressions for the CPs: ${ }^{15} u_{\alpha}$ $=\Gamma_{\alpha} / \Gamma, \quad u_{11}=-2\left(\Gamma_{1} \Gamma_{2} / \Gamma^{2}\right)\left(\delta \epsilon /|\Delta|^{2}\right)$, where $\delta \epsilon \equiv(E$ $\left.-E_{r}\right), \quad \Delta \equiv \delta \epsilon+i(\Gamma / 2), \quad \Gamma_{\alpha}$ is the decay width due to barrier $\alpha$, and $\Gamma=\Gamma_{1}+\Gamma_{2}$. Evaluating all the terms of Eq. (17) using these expressions, we derive

$$
\frac{d G_{111}(\omega=0) / d \omega}{G_{111}(\omega=0)}=i \omega \frac{\Gamma}{2}\left(\frac{1}{|\Delta|^{2}}-\frac{1}{\Gamma^{2}}\right),
$$

where $G_{111}(\omega=0)$ is the second-order weakly nonlinear dc conductance. ${ }^{2}$ This result is very interesting: it suggests that by measuring the ratio of the nonlinear conductances one obtains the microscopics of a tunneling device.

The above three examples demonstrate the power of the present theoretical formalism: it is suitable for analyzing weakly nonlinear dc and ac transport of mesoscopic conductors systematically. The expressions (15) and (17) are general to all orders of $\omega$. Setting $\omega=0$ they recover exactly those of SMT, ${ }^{2}$ thus providing the connection between SMT and the response theory at the weakly nonlinear ac level. Central to the theory is the self-consistent coupling of the quantum-mechanical equation with the Maxwell equations, thus the internal response of the system is taken into account, which is crucial to obtain electric current conservation and gauge invariance. Conceptually, we have introduced the multiple indexed, frequency-dependent characteristic potentials (CP and CVP) and the local density of states. We have also extended the concept of nonequilibrium charge distribution to the nonlinear order. These physical quantities are necessary in order to analyze weakly nonlinear ac response. For a mesoscopic conductor ${ }^{1}$ or even an atomic scale conductor, ${ }^{16}$ the scattering Green's function can be evaluated numerically thus our theoretical formalism provides the basis of numerical analysis for a variety of quantum conductors. In this paper, we have used the Lorentz gauge for electrodynamics. Clearly any gauge should work as is required by the Maxwell equations. Finally, we comment that our response theory is appropriate to equilibrium or near-equilibrium properties. For far-from-equilibrium problems one may employ the nonequilibrium Green's function ${ }^{17}$ for proper analysis.

We gratefully acknowledge financial support by the RGC of the SAR Government of Hong Kong under Grant No. HKU 7112/97P, by the NSF of China, the NSERC of Canada, and the FCAR of Quebec.
${ }^{1}$ See, for example, S. Datta, Electronic Transport in Mesoscopic Conductors (Cambridge University Press, New York, 1995).

${ }^{2}$ For a review, see M. Büttiker and T. Christen, in Mesoscopic Electron Transport, Vol. 345 of NATO Advanced Study Institute, Series E: Applied Science, edited by L. L. Sohn, L. P. Kouwenhoven, and G. Schoen (Kluwer Academic Publishers, Dordrecht, 1997), p. 259.

${ }^{3}$ M. Büttiker, J. Phys.: Condens. Matter 5, 9361 (1993).

${ }^{4}$ Ya. M. Blanter, F. W. J. Hekking, and M. Büttiker, Phys. Rev. Lett. 81, 1925 (1998); Ya. M. Blanter and M. Büttiker, Europhys. Lett. 42, 535 (1998).

${ }^{5}$ J. Wang and H. Guo, Phys. Rev. B 55, R11 090 (1996); J. Wang, Q. R. Zheng, and H. Guo, ibid. 55, 9763 (1997); 55, 9770 (1997).

${ }^{6}$ D. S. Fisher and P. A. Lee, Phys. Rev. B 23, 6851 (1981).

${ }^{7}$ H. U. Baranger and A. D. Stone, Phys. Rev. B 40, 8169 (1989); A. D. Stone and A. Szafer, IBM J. Res. Dev. 32, 384 (1988).

${ }^{8}$ P. G. N. de Vegvar, Phys. Rev. Lett. 70, 837 (1993).
${ }^{9}$ For a typical semiconductor quantum dot, the upper limit for the frequency can be 10 THz. See M. Büttiker, Phys. Rev. B 46, 12 485 (1992).

${ }^{10}$ J. Callaway, Quantum Theory of the Solid State, 2nd ed. (Academic Press, New York, 1991), Chap. 6.

${ }^{11}$ I. B. Levinson, Zh. Éksp. Teor. Fiz. 95, 2175 (1989) [Sov. Phys. JETP 68, 1257 (1989)].

${ }^{12}$ It can be shown that the induced vector potential will not appear in Eq. (13) when $\omega=0$.

${ }^{13}$ R. C. Ashoori, et al., Phys. Rev. Lett. 68, 3088 (1992); W. Chen et al., ibid. 73, 146 (1994).

${ }^{14}$ T. Christen and M. Büttiker, Phys. Rev. Lett. 77, 143 (1996).

${ }^{15}$ For simplicity, we have neglected the contribution from the induced vector potential.

${ }^{16}$ C. C. Wan et al., Appl. Phys. Lett. 71, 419 (1997).

${ }^{17}$ A. Jauho, N. S. Wingreen, and Y. Meir, Phys. Rev. B 50, 5528 (1994). 